\title{
Parathyroid Adenoma as a Rare Cause of Persistent Hypercalcemia in a Female with Polycythemia Vera
}

\author{
Ahmed M. Abdalhadi Mohamed A. Yassin \\ Hamad Medical Corporation, Doha, Qatar
}

\section{Keywords}

Polycythemia vera $\cdot$ Myeloproliferative neoplasms $\cdot$ Hypercalcemia $\cdot$ Primary

hyperparathyroidism · Parathyroid adenoma

\begin{abstract}
Polycythemia vera is one of the myeloproliferative neoplasms that is distinguished by the uncontrolled production of blood cells and an increased red cell mass due to acquired JAK2 mutation. It has many complications and it might increase the risk of other tumors. However, it does not cause hypercalcemia and is rarely associated with parathyroid adenoma. Here, we report on a 64-year-old female with polycythemia vera found to have hypercalcemia due to parathyroid adenoma.

\section{Introduction}

Polycythemia vera (PV) is one of the myeloproliferative neoplasm (MPN) subcategories which is characterized by the presence of a mutated JAK2 exon 14 or 12 that results in an elevated red blood cell production and panmyelosis in the bone marrow. It is a rare disease with an annual incidence of 2-3 per 100,000, and patients' median age is 65 years [1].

There was an unclear association between MPNs and solid tumors, but recently it has been shown, in some studies, that the risk of developing solid tumors is two times higher than in the general population, especially stomach, prostate, and lung cancer [2].

Hypercalcemia is a known complication of solid tumors such as lung and breast cancers, and of hematological malignancies such as lymphoma and multiple myeloma, but it is rarely associated with MPNs. However, it has been reported in very few cases [3]. PV does not cause hypercalcemia per se, so other causes should be thought of. 
Primary hyperparathyroidism due to parathyroid adenoma or carcinoma is a common cause of hypercalcemia; however, the relationship between PV and primary hyperparathyroidism is still obscure, and more case reports and studies are needed to clarify on this coexistence.

We report a case of primary hyperparathyroidism due to parathyroid adenoma as a cause of chronic hypercalcemia in a 64-year-old female who is known to have PV. To the best of our knowledge, this is the first case report of PV, diagnosed based on WHO criteria, to be associated with hypercalcemia due to parathyroid adenoma.

\section{Case Presentation}

A 64-year-old female was known to have PV since 2010. She fulfilled the 2008 WHO criteria [4], i.e., she had a hemoglobin level of $16.7 \mathrm{~g} / \mathrm{dL}$ (normal range 12-15), was positive for $J A K 2 \mathrm{~V} 617 \mathrm{~F}$, and had peripheral blood and bone marrow findings consistent with MPN. She was treated with hydroxyurea and frequent phlebotomies, when required, to maintain her hematocrit below $42 \%$. She presented to the clinic with a history of fatigue, generalized bone ache, and constipation for 4 months. There was no abdominal pain, polydipsia, polyuria, changes in her mood, or history of kidney stones. She was not taking any other medications apart from hydroxyurea.

Her general physical examination was normal, a head and neck examination was also normal, there were no palpable masses or nodules, and chest, heart and abdominal examinations were unremarkable as well.

The latest hemoglobin level was $12.8 \mathrm{~g} / \mathrm{dL}$ (normal range 12-15), her hematocrit was $37.3 \%$ (normal range 36-46), and her kidney and liver functions were normal. The blood calcium level was $2.73 \mathrm{mmol} / \mathrm{L}$ (normal range 2.15-2.50), the corrected calcium level was $2.87 \mathrm{mmol} / \mathrm{L}$ (normal range 2.20-2.55), 24-h urine calcium was normal, and the parathyroid hormone level was $183 \mathrm{pg} / \mathrm{mL}$ (normal range 15-65). Her previous results also showed hypercalcemia.

A neck ultrasound showed a normal-sized thyroid gland with a cystic nodule in the right and heterogeneous nodules in the left lobe, and two hypoechoic nodes were suspected in the region of both parathyroid glands. However, parathyroid glands cannot be visualized. There was no evidence of cervical lymphadenopathy. An abdominal ultrasound showed slight splenomegaly. Severe osteoporosis was confirmed by dual-energy X-ray absorptiometry scanning. A parathyroid MIBI scan was negative for enlarged overactive parathyroid adenoma or hyperplasia. However, parathyroid SPECT CT showed a hyperfunctioning parathyroid adenoma in the left-upper-pole thyroid region (Fig. 1,2).

The patient was followed up by the endocrinologist, who started her on cinacalcet and referred her to the surgical team for parathyroidectomy.

\section{Discussion}

Polycythemia is defined as an increased red blood cell mass and production which causes an elevation in hemoglobin and hematocrit. There are two types of absolute polycythemia: primary and secondary polycythemia. Secondary polycythemia is a result of other conditions which increase erythropoietin production. It is usually associated with solid tumors, such as renal cell carcinoma, hepatoma, and ovarian cancer. Also, it is a bodily response to chronic hypoxemia. In contrast, primary polycythemia is caused by bone marrow disorders, which mainly leads to abnormal erythroid cell line proliferation. PV is distinguished from other 
Fig. 1. Asymmetrical tracer uptake; focally increased uptake seen in the left upper pole.

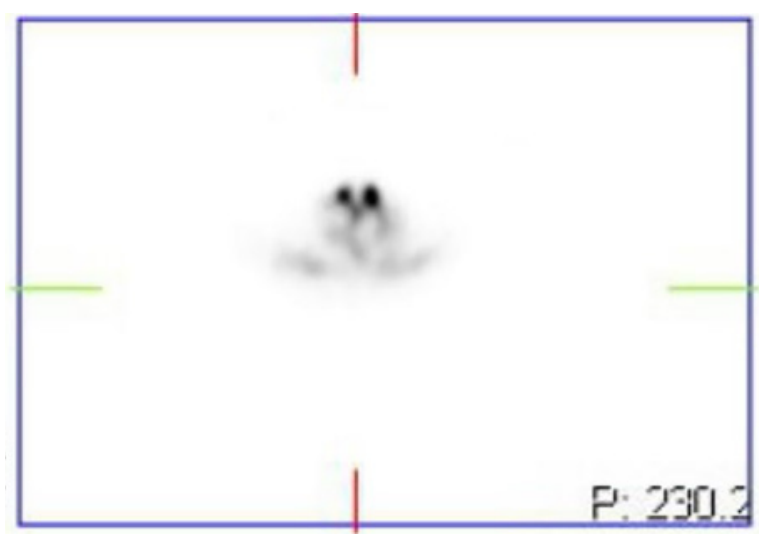

Fig. 2. One-hour SPECT CT image showing prominent MIBI retention in the left upper pole.

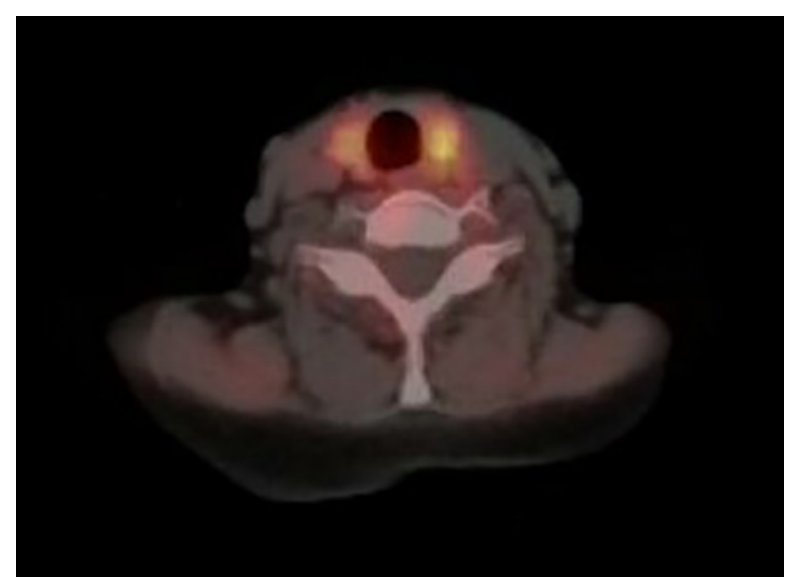

types by higher erythrocyte production than other blood cells and positive JAK2 mutation in almost all patients.

The WHO classifies MPNs into seven subcategories; one of them is PV. The 2016 WHO criteria for PV diagnosis include three major criteria and one minor criterion. The major criteria are: hemoglobin $>16.5 \mathrm{~g} / \mathrm{dL}$ and $>16.0 \mathrm{~g} / \mathrm{dL}$ in men and women, respectively, or hematocrit $>49 \%$ (men)/>48\% (women), or an increased red cell mass; bone marrow biopsy showing hypercellularity for age with trilineage growth (panmyelosis) including prominent erythroid, granulocytic, and megakaryocytic proliferation with pleomorphic, mature megakaryocytes (differences in size); and the presence of JAK2 or JAK2 exon 12 mutation. A subnormal serum erythropoietin level is the minor criterion. For a PV diagnosis, either all major criteria are required or the first two major criteria plus a subnormal serum erythropoietin level [5].

PV has some complications, which include thrombosis, bleeding, hepatosplenomegaly, erythromelalgia, hyperuricemia, myelofibrosis, and transformation to myelodysplasia or acute leukemia. However, hypercalcemia is not one of these complications. Hypercalcemia, which occurs in multiple myeloma, lymphoma, or any other solid tumor, is usually related to the disease itself, but in PV, it is usually due to other causes such as primary hyperparathyroidism, due to adenoma or carcinoma, or due to other solid malignancies. Also, it has been reported that hypercalcemia is associated with PV transformation into acute myeloid leukemia [6]. Although there are case reports and studies that describe an association between hypercalcemia and PV, most of them are old, from a time at which the PV diagnosis was unclear, and it was established before introducing the WHO criteria in 2008 [7]. 
Parathyroid adenoma could be the cause of hypercalcemia in PV, and it is mentioned in one study that it can increase the risk of PV, but this study included patients from 1958 to 2008 and the method of diagnosis was unexplained [8]. Also, it was shown in a case report that PV and hypercalcemia have improved after thyroidectomy, but in that case, JAK2 was negative and the method of diagnosis was not available [9]. On the other hand, some studies have shown that hyperparathyroidism increases red cell destruction in uremic patients by increasing the calcium level inside them [10].

Based on the available case reports and studies, we could not conclude that there is an association between hyperparathyroidism and PV; also, most of these studies were done before the introduction of the WHO diagnostic criteria, so we need more studies to understand completely if it is just a coexistence or a cause-and-effect relationship.

Due to the relative rarity of PV and the unknown association between hypercalcemia and PV, the diagnosis was delayed. Physicians should be aware of this possible association in order to avoid missing or delaying the diagnosis in similar cases and to consider improving the quality of life of those patients [11].

Finally, we can say that our case could be the first case with a well-established PV diagnosis, based on the 2008 WHO criteria, diagnosed with hypercalcemia due to parathyroid adenoma.

\section{Conclusions}

Although an association between hypercalcemia and some solid and hematological malignancies has been reported in many studies, it is not well reported for PV cases, especially after the introduction of the WHO criteria. Parathyroid adenoma could be the cause of hypercalcemia in PV. We would like to highlight this rare finding in order to increase physicians' awareness, and to encourage them to report similar cases to understand this association.

\section{Acknowledgments}

We acknowledge the internal medicine residency program for scientific support and Dr. Yasmin Tolymat (endocrinologist) for clinical care.

\section{Statement of Ethics}

The case was approved by the Hamad Medical Corporation Medical Research Center, and the patient signed a written informed consent to publish her case (including publication of images).

\section{Disclosure Statement}

The authors report no conflicts of interest regarding this work. 


\section{Funding Sources}

The case report was funded by the Qatar National Library.

\section{Author Contributions}

Both authors contributed equally to manuscript writing and editing.

\section{References}

1 Yassin MA, Taher A, Mathews V, Hou HA, Shamsi T, Tuğlular TF, et al. MERGE: a multinational, multicenter observational registry for myeloproliferative neoplasms in Asia, including Middle East, Turkey, and Algeria. Cancer Med. 2020 [Online ahead of print].

2 Hong J, Lee DY, Byun JM, Lee JY, Koh Y, Shin DY, et al. Risk of disease transformation and second primary solid tumors in patients with myeloproliferative neoplasms. Blood Adv. 2019;3(22):3700-8.

3 Khoury N, Chang J, Gru AA, Whyte MP. Resorptive hypercalcemia in post-essential thrombocythemia myelofibrosis: treatment with denosumab. J Clin Endocrinol Metab. 2012;97(9):3051-5.

4 Thiele J, Kvasnicka HM. The 2008 WHO diagnostic criteria for polycythemia vera, essential thrombocythemia, and primary myelofibrosis. Curr Hematol Malig Rep. 2009;4(1):33-40.

5 Barbui T, Thiele J, Gisslinger H, Kvasnicka HM, Vannucchi AM, Guglielmelli P, et al. The 2016 WHO classification and diagnostic criteria for myeloproliferative neoplasms: document summary and in-depth discussion. Blood Cancer J. 2018;8(2):15.

6 Chambers I, Truong P, Kallail KJ, Palko W. Extensive bone marrow necrosis and osteolytic lesions in a case of acute myeloid leukemia transformed from polycythemia vera. Cureus. 2016;8(6):e639.

7 Brown GE, Roth GM. The reduction of hypercalcemia in cases of polycythemia vera by phenylhydrazine. J Clin Invest. 1928;6(1):159-69.

8 Fallah M, Kharazmi E, Sundquist J, Hemminki K. Nonendocrine cancers associated with benign and malignant parathyroid tumors. J Clin Endocrinol Metab. 2011;96(7):E1108-14.

9 Kulaylat AN, Jung EE, Saunders BD. The role of parathyroidectomy in JAK2 mutation negative polycythemia vera. Int J Hematol. 2014;100(6):615-8.

10 Bogin E, Massry SG, Levi J, Djaldeti M, Bristol G, Smith J. Effect of parathyroid hormone on osmotic fragility of human erythrocytes. J Clin Invest. 1982;69(4):1017-25.

11 Taher AT, Yassin MA, Xiao Z, Hou HA, Tuglular T, Mathews V, et al. Impact of myeloproliferative neoplasms (MPNs) on health-related quality of life (HRQOL) and medical resource utilization: results from the MERGE registry. Blood. 2018;132(Suppl 1):4311. 